CERN - PS DIVISION

CERN/PS 2002-077 (AE)

\title{
MULTITURN EXTRACTION: PERFORMANCE ANALYSIS OF OLD AND NEW APPROACHES
}

\author{
R. Cappi, M. Giovannozzi
}

\begin{abstract}
For some applications an intermediate extraction mode between fast (one turn) and slow (some hundreds to many billions turns) is needed. This is the case of the five-turn extraction used to transfer the proton beam from the CERN Proton Synchrotron to the Super Proton Synchrotron. The present approach is based on beam slicing via an electrostatic septum. However, recently a novel technique was proposed. By using nonlinear magnetic elements, stable islands can be generated in the transverse phase space. Furthermore, if the linear tune is varied slowly, it is possible to trap the charged particles inside the stable islands so that the beam is split into different beamlets ready for extraction. In this paper, both techniques are discussed in details with special emphasis on their robustness and the phase space matching of the extracted beam.
\end{abstract}

2002 Charged Particle Optics Conference, Greenbelt MD (USA) October 22 - 25, 2002

Geneva, Switzerland

October 29, 2002 


\section{Introduction}

Charged particles can be extracted from a circular machine by two different methods: i) fast extraction, ii) slow extraction. In the first method, the whole beam is ejected from the machine in one turn by means of fast dipole (kicker) and septum magnets. This technique can be used for transferring the beam either to a subsequent machine or towards a target for physics experiments. The second method, is based on the effect of a third-order resonance $[1,2]$ : unstable motion generated by the separatrix joining the three unstable fixed points increases the particles' amplitude. This technique allows beam extraction over many machine turns (typically many millions) and it is only used when delivering beam to a target for physics experiments. For the sake of completeness, one should also mention the so-called fast resonant or half-integer extraction, where the beam is extracted by means of the half-integer resonance over some hundreds turns (see Ref. [4] for the pioneering work on the half-integer stop-bands and Refs. [5, 6] for the KEK, Refs. [7, 8] for CERN Super Proton Synchrotron (SPS), and Ref. [9] for the FermiLab main ring studies, respectively).

The two approaches, fast and slow extraction, differ not only in the time-distribution of the extracted beam, but also in the effect on the extracted beam emittance. In fact, fast extraction leaves unchanged the beam emittance of the circulating beam, while slowextraction reduces the horizontal beam emittance to virtually zero due to the beam stretching along the unstable separatrix (see Ref. [3] and references therein for a detailed discussion of this issue), the vertical one being unchanged.

In some special cases, an intermediate extraction mode, called multi-turn extraction, is needed. This is the case of the transfer between the CERN Proton Synchrotron (PS) and the SPS. The two machines have different circumferences, satisfying the relation $C_{S P S}=11 C_{P S}$. Hence, to fill the SPS completely, requires ten fast-extracted pulses from the PS (the empty gap in the SPS is needed for the rise-time of the injection kicker). If the filling time has to be minimised, then the solution consists of extracting the beam over a few turns, instead of a single one. In practise, a five-turn extraction was proposed and used since many years, allowing the SPS to be filled with only two PS pulses. Such an approach is called Continuous Transfer (CT) [10]. In the design of this extraction mode a second constraint has been taken into account, namely the necessity of reducing the horizontal transverse emittance of the extracted beam to overcome the acceptance limitation of the SPS machine ${ }^{1)}$.

For this, the beam is sliced by means of an electrostatic septum: by setting the horizontal tune of the machine to the value 6.25 it is possible to generate exactly five slices (the details can be found in the next sections). A number of drawbacks are present, though: i) beam losses, especially at the electrostatic septum, are an intrinsic and unavoidable characteristic of this extraction process. They amount to about $10-15 \%$ of the total beam intensity; ii) the extracted slices do not match the natural partitioning of phase space into circles. This generates a betatron mismatch, i.e. each beam slice has different equivalent horizontal optical parameters which means that a non negligible emittance blow-up at injection in the SPS has to be expected. Furthermore, this blow-up will be different for each slice.

Although this extraction mode is the best solution presently in operation, it may

1) In fact, the acceptance limitation in the SPS machine is in the vertical plane, but a special optics in the transfer line joining the PS and SPS allows exchanging the two transverse planes (in particular the emittance values) [11]. 
represent a hard limit for future very high-intensity proton beams such as those for the planned CERN Neutrino to Gran Sasso (CNGS) beam [12]. This is a high-intensity proton beam: in its nominal version, the PS should deliver two pulses of more than $3 \times 10^{13}$ protons each to the SPS, an intensity close to the present PS record intensity. To increase the rate of good events for physics, thus reducing the data-taking time, efforts are being made to study a possible intensity upgrade by a factor of about two [13]. In the new scenario, the strong beam losses related to the present scheme of the five-turn CT would not be acceptable, and the properties of the extracted beam (matching of phase space structure and transverse emittance) would not allow an efficient injection into the SPS.

Recently a novel method has been proposed $[14,15]$ : it is based on adiabatic capture of charged particles in stable islands of transverse phase space. The approach consists in generating stable islands in the transverse phase space occupied by the beam, by means of special magnetic elements such as sextupoles and octupoles, and to move the islands by slowly-changing the tune of the machine. Numerical simulations are quite encouraging (see next sections for more details), and an intense experimental activity has been undertaken at the PS machine to assess the feasibility of this new method. The main advantage over the present CT extraction mode is that the beam is split by the action of stable islands sweeping through the central region of phase space. Hence no intercepting device is used. Furthermore, the resulting beamlets are better matched to the phase space topology.

The outline of the paper is the following: in section 2 the main definitions concerning the beam emittance, the computation of the betatron mismatch, and Twiss parameters, as well as the distributions used in the analytical computations are presented. In section 3 the present CT extraction mode is described in details including the issue of phase space matching of the different slices (section 3.2). In section 4 the proposed multi-turn extraction based on island trapping is discussed and the results of numerical simulations presented. Furthermore, the properties of phase space matching (section 4.3) together with the robustness of the method against variation of the strength of the nonlinear elements is discussed. Finally, conclusions are drawn in section 5.

\section{Betatron Mismatch in Normalised Phase Space}

\subsection{Definition of Mismatch Parameters}

When a beam is injected into a circular machine, it may experience emittance blowup due to a number of different phenomena. In this paper we will focus on the betatron mismatch $[16,17]$, which occurs when the beam optical parameters at injection do not match those of the circular machine. In this case, tails may grow at the expense of the beam core, thus increasing the overall beam emittance. Of course, such a behaviour is particularly harmful for high-intensity beams as they usually fill completely the machine acceptance.

To quantify the betatron mismatch, it is customary to start from the statistical definition of beam emittance $\epsilon$ and optical parameters $\bar{\alpha}, \bar{\beta}, \bar{\gamma}[18]$ in terms of the second order moments of the beam distribution [19]. By using the notation $\left(x, x^{\prime}\right)$ for the physical phase space co-ordinates, while $\left(\hat{x}, \hat{x}^{\prime}\right)$ represent normalised phase space co-ordinates, the following holds:

$$
\begin{aligned}
<x^{2}> & =\bar{\beta} \epsilon \\
<x x^{\prime}> & =-\bar{\alpha} \epsilon \\
<x^{\prime 2}> & =\bar{\gamma} \epsilon .
\end{aligned}
$$


In the equations quoted above the symbol $\langle\cdot\rangle$ stands for the average over the beam distribution of the specified variable. This approach is equivalent to fit an ellipse to the phase space distribution, where the fit parameters are its surface, amplitude, and orientation.

If the nominal Twiss parameters $\alpha, \beta, \gamma$, i.e. those relative to the nominal optics, are used to transform Eq. (1) into normalised phase space via the well-known, transformation rules $[18]$

$$
\hat{x}=\frac{x}{\sqrt{\beta}} \quad \hat{x}^{\prime}=\frac{\alpha}{\sqrt{\beta}} x+\sqrt{\beta} x^{\prime}
$$

one obtains the key relations:

$$
\begin{aligned}
<\hat{x}^{2}> & =\frac{\bar{\beta}}{\beta} \epsilon \\
<\hat{x} \hat{x}^{\prime}> & =\left(\alpha \frac{\bar{\beta}}{\beta}-\bar{\alpha}\right) \epsilon \\
<\hat{x}^{\prime 2}> & =\left(\alpha^{2} \frac{\bar{\beta}}{\beta}-2 \alpha \bar{\alpha}+\beta \bar{\gamma}\right) \epsilon .
\end{aligned}
$$

The value of the emittance can be obtained by solving Eqs. (3), namely

$$
\epsilon=<\hat{x}^{2}><\hat{x}^{\prime 2}>-<\hat{x} \hat{x}^{\prime}>^{2} .
$$

The meaning of the coefficients $\bar{\beta} / \beta$ and $\alpha \bar{\beta} / \beta-\bar{\alpha}$ is clear: they measure the departure of the $1 \sigma$ contour of the beam distribution from the circular shape it should have in normalised phase space. Furthermore, it turns out that the emittance after filamentation $\epsilon_{\text {after fil. }}$, i.e. when the circular symmetry has been restored, is exactly expressed as

$$
\epsilon_{\text {after fil. }}=H \epsilon, \quad \text { where } \quad H=\frac{1}{2}\left[\frac{\bar{\beta}}{\beta}+\frac{\beta}{\bar{\beta}}+\left(\alpha \sqrt{\frac{\bar{\beta}}{\beta}}-\bar{\alpha} \sqrt{\frac{\beta}{\bar{\beta}}}\right)^{2}\right],
$$

where $\epsilon$ is the initial value of the beam emittance.

\subsection{Beam Distribution for the Present CT}

It is customary to assume that the transverse beam distribution is a Gaussian function. Under this assumption it is possible to derive all the relevant quantities to describe the betatron mismatch of the different slices of the present CT in closed form [20]. However, the presence of long, and strictly speaking infinite, tails makes some results difficult to interpret (see Ref. [20] for more details on this subject). For this reason, the choice for the beam distribution has been changed and a quasi-parabolic distribution [21, $22,23]$ has been used in the computations presented in this paper.

A quasi-parabolic distribution is represented by a smooth function

$$
\rho_{\mathrm{P}}\left(\hat{x}, \hat{x}^{\prime}, m\right)=\left\{\begin{array}{lll}
\frac{m+1}{2 \pi \epsilon(m+2)}\left[1-\frac{\hat{x}^{2}+\hat{x}^{\prime 2}}{2 \epsilon(m+2)}\right]^{m} & \text { if } & \hat{x}^{2}+\hat{x}^{\prime 2} \leq 2 \epsilon(m+2), \\
0 & \text { if } & \hat{x}^{2}+\hat{x}^{\prime 2}>2 \epsilon(m+2) .
\end{array}\right.
$$




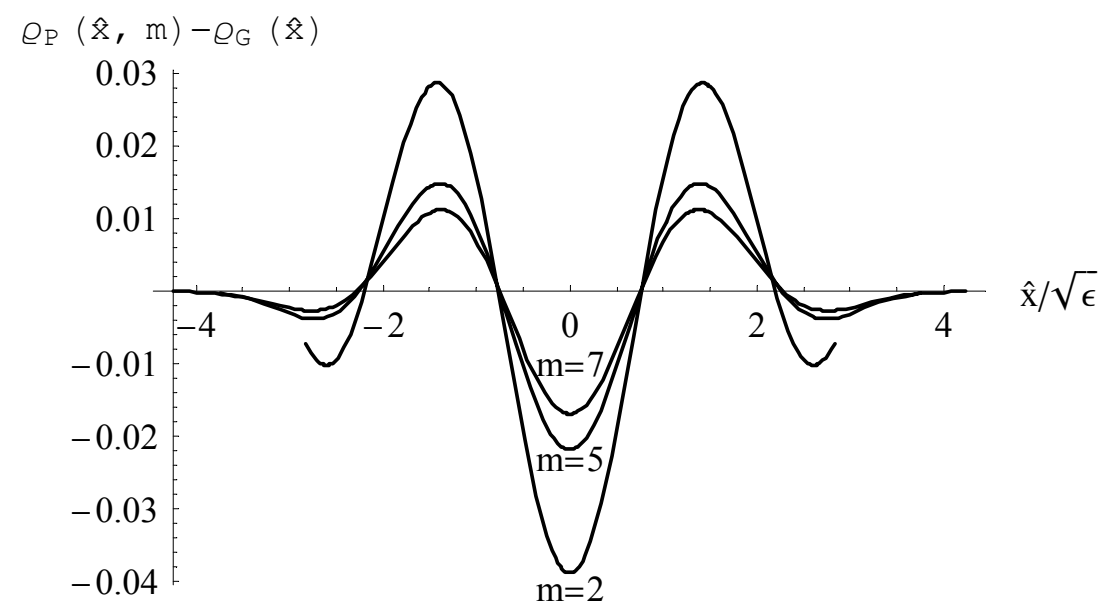

Figure 1: Comparison between Gaussian distribution function $\rho_{\mathrm{G}}$ and a quasi-parabolic one $\rho_{\mathrm{P}}$ (integration over the angular variable $\hat{x}^{\prime}$ has been performed). The difference between the two distributions is plotted as a function of $\hat{x} / \sqrt{\epsilon}$ for different values of $m$.

where $m$ is a parameter and $\epsilon$ the total beam emittance. Such a distribution function has no tails, making it a good model for a real beam. In all the computations presented in this paper $m=5$ has been used. In Fig. 1 the comparison between a Gaussian distribution $\rho_{\mathrm{G}}$ and a quasi-parabolic one $\rho_{\mathrm{P}}$ is shown as a function of the parameter $m$. It is clearly seen that the difference between the quasi-parabolic distributions and the Gaussian is rather small, thus making it an ideal choice for the study presented in this paper.

\section{$3 \quad$ Present Continuous Transfer}

\subsection{Basic Principle}

The principle of the present CT extraction is quite simple [10]: just before extraction, the horizontal tune $Q_{H}$ of the PS machine is set to the value 6.25. At the same time the closed orbit is modified so that the blade of an electrostatic septum intercepts the beam. Then, because of the value of the horizontal tune, four slices are shaved off the main core and extracted as a continuous ribbon over four turns. After that, only the central core remains in the machine and it is extracted last, during the fifth turn, by changing the beam trajectory, so as to jump over the septum blade. In addition to the electrostatic septum used to slice the beam, a kicker and a magnetic septum are used for the extraction. In Fig. 2 the layout of the extraction elements is shown together with the horizontal normalised phase space. It should be apparent that the average position and angle differ for each slice, therefore, in practice, the beam trajectory has to be different for each slice. This is obtained by means of dedicated fast dipoles.

\subsection{Transverse Properties of the Extracted Beam}

As already mentioned in the Introduction, the extracted beam has a smaller horizontal emittance than the circulating one. However, the five slices have different shapes, which means different emittances and optical parameters at extraction.

By applying the approach described in section 2, it is possible to obtain the mismatch factors and the emittance of the different slices of the present CT. The detailed computations can be found in Ref. [20]. In this paper we will report the evaluation for a simplified case. The relative position between the blade of the electrostatic septum and the 

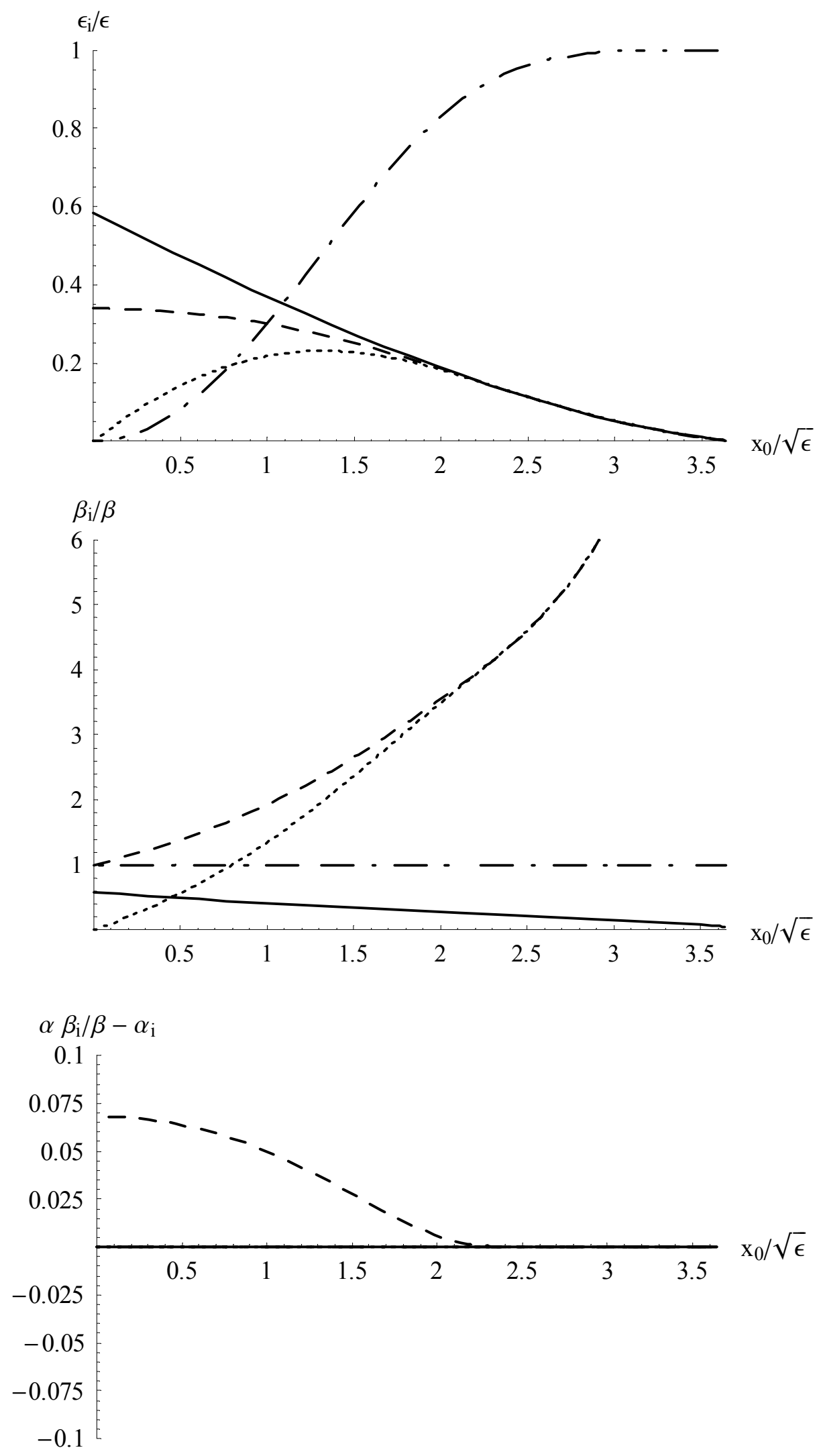

Figure 3: Beam emittance $\epsilon_{i} / \epsilon$ (upper), mismatch factor $\bar{\beta}_{i} / \beta$ (centre), and $\alpha \bar{\beta}_{i} / \beta-\bar{\alpha}_{i}$ (lower) for the different slices as a function of $\hat{x}_{0} / \sqrt{\epsilon}$, representing the position of the electrostatic septum blade in units of beam emittance (first slice solid, second dashed, fourth dotted, fifth dot-dashed). 
without losses.

Numerical simulations were carried out to study this novel approach $[14,15]$. In the model, only the horizontal betatron motion is taken into account, as the vertical one can be safely neglected for our purpose. The nonlinear magnets are assumed to be at the same location in the ring and are represented in the single-kick approximation (see Ref. [24] for more details). Under these assumptions, the one-turn transfer map can be expressed as $\hat{\mathbf{X}}_{n+1}=\mathbf{M}_{n}\left(\hat{\mathbf{X}}_{n}\right)$ :

$$
\left(\begin{array}{c}
\hat{X}_{n+1} \\
\hat{X}_{n+1}^{\prime}
\end{array}\right)=R\left(2 \pi \nu_{n}\right)\left(\begin{array}{c}
\hat{X}_{n} \\
\hat{X}_{n}^{\prime}+\hat{X}_{n}^{2}+\kappa \hat{X}_{n}^{3}
\end{array}\right)
$$

where $\left(\hat{X}, \hat{X}^{\prime}\right)$ are obtained from the Courant-Snyder [18] co-ordinates $\left(\hat{x}, \hat{x}^{\prime}\right)$ by means of the non-symplectic transformations

$$
\hat{X}=\frac{1}{2} K_{2} \beta_{H}^{3 / 2} \hat{x} \quad \hat{X}^{\prime}=\frac{1}{2} K_{2} \beta_{H}^{3 / 2} \hat{x}^{\prime}, \quad \text { with } \quad K_{l}=\frac{L}{B_{0} \rho} \frac{\partial^{l} B_{y}}{\partial x^{l}},
$$

$K_{2}\left(K_{3}\right)$ being the integrated sextupole (octupole) gradient, $L$ the length of the nonlinear element, $B_{y}$ the vertical component of the magnetic field, $B_{0} \rho$ the magnetic rigidity of the charged particle, and $\beta_{H}$ the value of the horizontal beta-function at the nonlinear elements location. $R\left(2 \pi \nu_{n}\right)$ is a $2 \times 2$ rotation matrix of angle $\nu_{n}$ (the fractional part of $\left.Q_{H}\right)$

$$
R\left(2 \pi \nu_{n}\right)=\left(\begin{array}{rr}
\cos 2 \pi \nu_{n} & \sin 2 \pi \nu_{n} \\
-\sin 2 \pi \nu_{n} & \cos 2 \pi \nu_{n}
\end{array}\right)
$$

and $\kappa$ is expressed as

$$
\kappa=\frac{2}{3} \frac{K_{3}}{\beta_{H} K_{2}^{2}}
$$

The map (7) is actually a time-dependent system as the linear tune is time-dependent. The importance of introducing a time-dependence is twofold. Firstly, it allows varying the phase space topology, thus creating and moving the islands. Secondly, it allows trapping particles inside the islands, which is the necessary condition for the proposed scheme to work efficiently. The time-dependence breaks the invariance of the separatrix around the stable islands, thus making it permeable for the particles. In a time-independent system a particle outside an island would never be able to jump across the separatrix, as this would mean changing the value of the invariant of motion. However, a slow variation of the linear tune, adiabatic with respect to the time scale introduced by the betatron oscillations, allows particles to cross the separatix and to be trapped inside the newly-created islands.

A good choice for the time-dependence of the tune variation is shown in Fig. 4. Unless otherwise stated, the results of numerical simulations refer to such a function. In the first part, the linear tune is decreased linearly from its initial value of 0.252 to 0.249. During this part, the capture process takes place. Then a zero-slope part follows, used to allow the beam to filament after capture, to match better the phase space topology. Finally, a second linear decrease to the value 0.245 is performed which allows the islands to be moved towards higher amplitudes before extraction.

The different stages of this novel extraction are shown in Fig. 5, evaluated by the 
The whole process has been simulated numerically by using the model (7) with $\kappa=-1.6^{2)}$ and a set of Gaussian-distributed initial conditions, whose evolution under the dynamics induced by the system (7) is shown in Fig. 6. The Coulomb interaction between the charged particles is not taken into account. Preliminary estimates [14] seem to indicate that the space charge tune shift should not be a serious problem for the proposed approach. In fact, the capture is performed by trapping outer particles and they are less affected by the space charge tune shift than those particles in the beam core. However, further numerical simulations will be needed to study this delicate point.
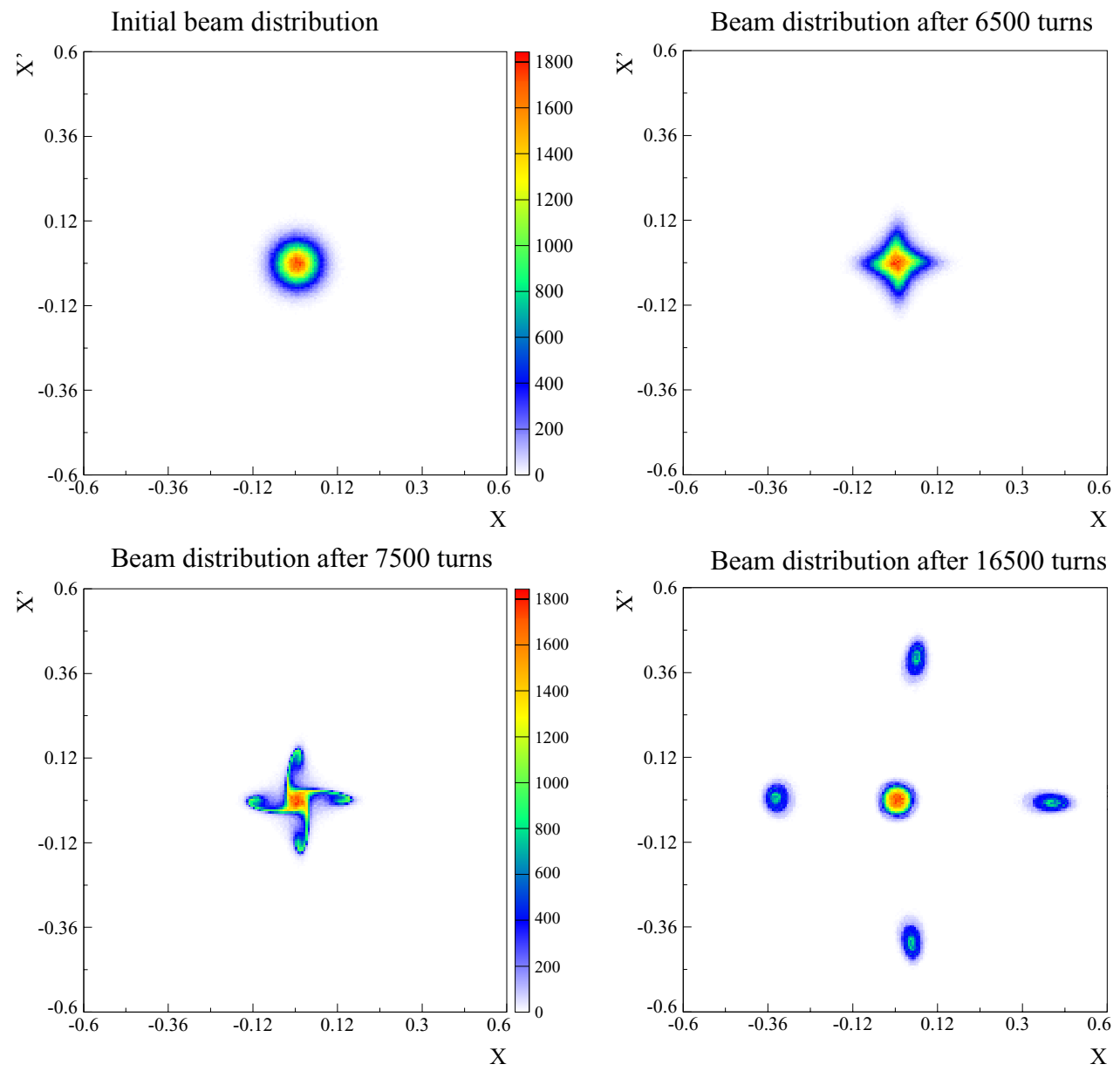

Figure 6: Evolution of the beam distribution during the trapping process with four islands. The different plots correspond to tune values represented by solid squares in Fig. 4. Each plot represents $4.9 \times 10^{5}$ points. The initial Gaussian distribution is centred on zero and has $\sigma=0.045$.

The trapping process is clearly visible: five well-separated beam slices are generated at the end of the process. No particles are lost during the trapping phase, nor when the islands are moved. Therefore, this extraction process is essentially lossless. The five slices have rather similar surfaces, but also their shape matches the phase space topology very well, making the five parts similar as far as transverse properties are concerned. It is

${ }^{2)}$ It is worth pointing out that the values of the multipolar coefficients $K_{2}$ and $K_{3}$ computed for a realistic case (PS machine) are well within technological limits, being of the order of $28 \mathrm{~T} / \mathrm{m}$ and $3000 \mathrm{~T} / \mathrm{m}^{2}$, for $K_{2}$ and $K_{3}$ respectively (see Ref. [26] for more details). 
worthwhile pointing out that the first four extracted beam slices have exactly the same emittance, as their shape is dictated by the same phase space structure, i.e. the island along the positive $\hat{X}$ axis. Also in this respect, the novel approach proves to be potentially superior to the present CT extraction mode.

The distribution functions $\rho(\hat{X})$ for the different beam slices at the end of the trapping and transport process are shown in Fig. 7. They are all Gaussian-like, as is the initial beam distribution, thus showing that the shape is almost preserved throughout the whole process. Still, further optimisation is required to improve the intensity sharing between the different beamlets, as the last one is much stronger than the others.

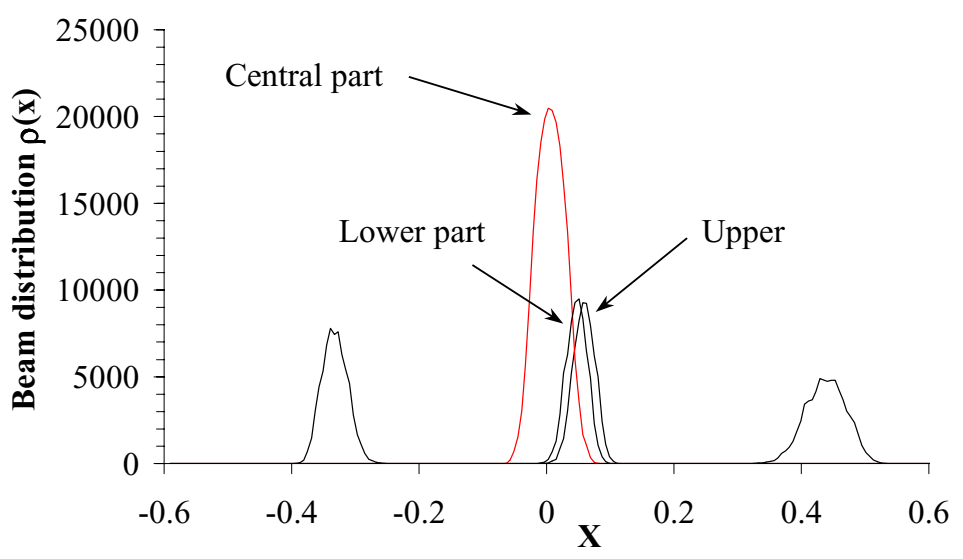

Figure 7: Beam distribution function $\rho(\hat{X})$ at the end of the capture and transport process, for all five beam slices shown in Fig 6 (lower right) for $\kappa=-1.6$. The central beamlet is clearly too much intense with respect to the others.

\subsection{Digression: Adiabatic Trapping Using Other Resonances}

It is interesting to point out that the proposed approach can work also for other cases [15], such as the third-order resonance. This resonance is inherently unstable [24], hence no closed curves surround the origin of phase space. This makes the adiabatic capture much more difficult, due to the risk of particle loss. In Fig. 8, the linear tune as a function of $n$ is shown.

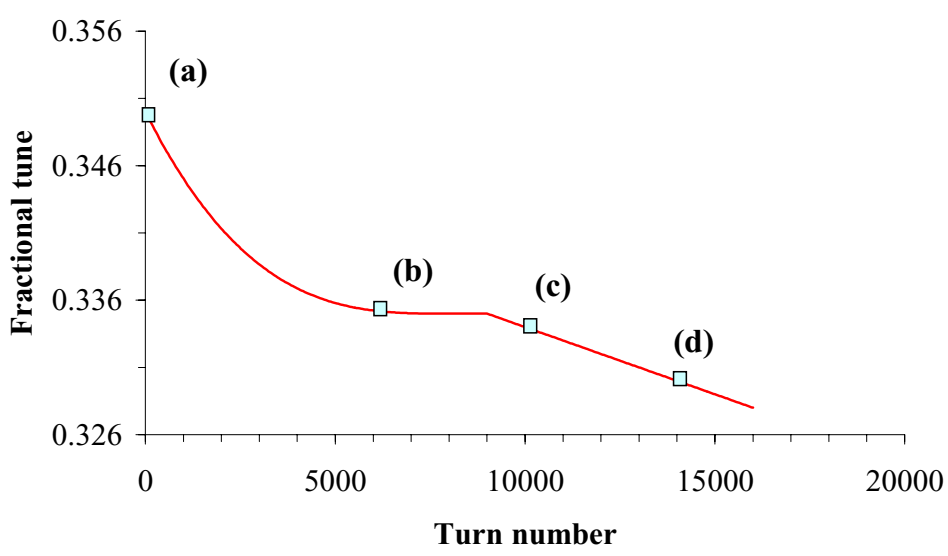

Figure 8: Linear tune $\nu$ as a function of turn number $n$ for the third-order resonance. The points on the curve labelled with $(\mathbf{a}),(\mathbf{b}),(\mathbf{c})$, and $(\mathbf{d})$ correspond to the values of $\nu_{n}$ used in Fig 9. 
In this case, to ensure a good trapping, a polynomial dependence of order three has been selected, instead of the plain linear decrease used for the fourth-order resonance.

The various steps of the trapping process are shown in Fig 9, obtained with the system (7) and $\kappa=-5$. In spite of the different topology, three well-separated slices can
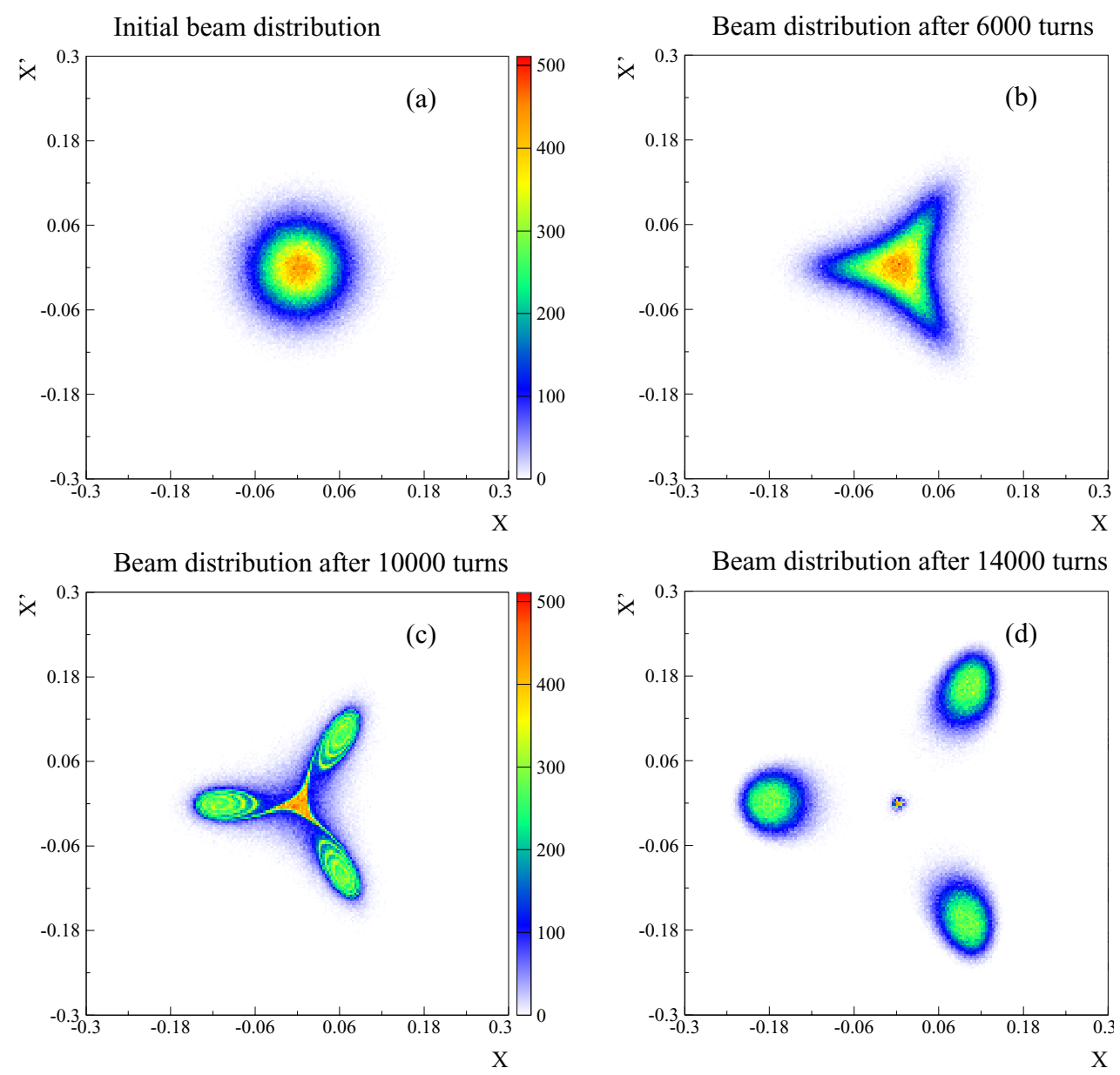

Figure 9: Evolution of the beam distribution during the trapping process with three islands. Each plot represents $4.9 \times 10^{5}$ points. The initial Gaussian distribution is centred on zero and has $\sigma=0.045$.

be found at the end of the process. No losses occur during the whole process and the region around the origin is almost emptied, apart from very few particles. Finally, the distribution $\rho(\hat{X})$ is shown in Fig. 10 .

\subsection{Transverse Properties of the Extracted Beam}

Similarly to what has been presented for the present CT, a parametric study of the mismatch parameters for the novel approach was performed. The first test concerns the dependence of the trapped beam parameters on the numerical value of $\kappa$. By means of numerical simulations and using the definitions in section 2 , the quantities $\bar{\beta}_{i} / \beta, \alpha \bar{\beta}_{i} / \beta-$ $\bar{\alpha}_{i}$, as well as the beam emittance $\epsilon_{i}$ have been evaluated. In all the cases considered, the tune dependence on the turn number is the one shown in Fig. 4. The results are shown in Fig. 11, where the modulus of $\kappa$ has been used as independent variable. The two series of points refer to the first four extracted beamlets (squares) and the fifth one (triangles). In all the cases shown here, only a mild dependence on $|\kappa|$ is visible. Around the nominal 

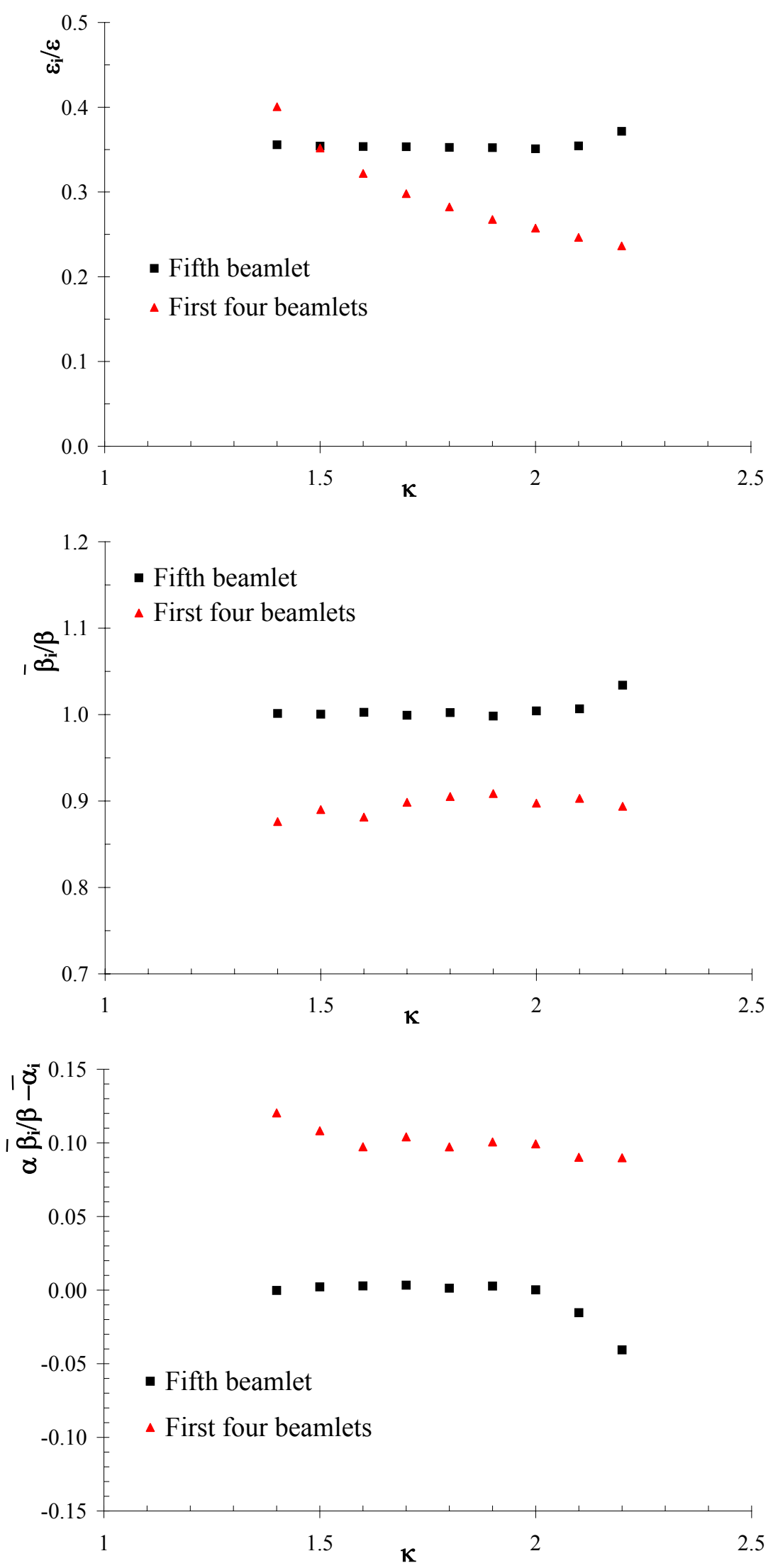

Figure 11: Beam emittance $\epsilon_{i} / \epsilon$ (upper), mismatch factor $\bar{\beta}_{i} / \beta$ (centre), and mismatch factor $\alpha \bar{\beta}_{i} / \beta-\bar{\alpha}_{i}$ (lower) for the four first beamlets (squares) and for the last one (triangles) as a function of $|\kappa|$ for the novel extraction mode based on adiabatic capture. 


\section{References}

[1] M. M. Gordon and T. A. Welton, Bull. Am. Phys. Soc. 3, 57 (1958).

[2] K. Hiramoto, M. Nishi, Nucl. Instrum. \& Methods A 322, 154 (1992).

[3] L. Bongini, A. Bazzani, G. Turchetti, and I. Hofmann, Phys. Rev. ST Accel. Beams, 4, 114201, (2001).

[4] D. Möhl, L. Thorndahl, Crossing of Half Integer Stop-Bands: an Experimental Study Performed on the CPS, CERN-ISR-300-LI-GS-69-20 (1969) (unpublished).

[5] K. Endo, C. Steinbach, Half Integer Resonant Extraction From Main Ring of KEK Proton Synchrotron, KEK-77-13 (1977) (unpublished).

[6] Y. Shoji, K. Marutsuka, T. Toyama, H. Sato, Low-emittance Slow Extraction Using Half-Integer Resonance, KEK-Preprint-95-99 (1995) (unpublished).

[7] K. H. Kissler, A. J. Riche, W. Scandale, G. Schröder, IEEE Trans. Nucl. Sci. 26, 3228 (1979).

[8] M. Gyr, E. B. Vossenberg, Half-Integer Fast Resonant Extraction with quasi Rectangular Spill, in Proceedings of the 3rd European Particle Accelerator Conference, edited by H. Henke et al. (Ed. Frontières, Gif-sur-Yvette, 1992), p. 1501.

[9] D. Trbojevic, M. Harrison, in Proceedings of the 1991 Particle Accelerator Conference, edited by J. Chew and L. Lizana (IEEE, New York, 1991), p. 96.

[10] C. Bovet et al., in Proceedings of the 1973 Particle Accelerator Conference, edited by D. W. Dupen (IEEE, New York, 1973), p. 438.

[11] L. R. Evans, A Phase Plane Exchange Section for the SPS Antiproton Injection Beam Line, CERN SPS (DI) 80-2, (1980) (unpublished).

[12] K. Elsener (Ed.) et al., CERN Yellow Report No. 98-02, (1998) (unpublished).

[13] R. Cappi (Ed.), K. Cornelis, J.-P. Delahaye, R. Garoby, H. Haseroth, K. Hübner, T. Linnecar, S. Myers, K. Schindl, C. Wyss, Increasing proton intensity of PS and SPS, CERN-PS (AE) 2001-041, (2001) (unpublished).

[14] R. Cappi, M. Giovannozzi, Phys. Rev. Lett. 88, 104801, (2002).

[15] R. Cappi, M. Giovannozzi, in Proceedings of 8th European Particle Accelerator Conference, edited by J. Poole and C. petit-Jean-Genaz (Institute of Physics UK, London, 2002), p. 1250.

[16] P. J. Bryant, K. Johnsen, Circular Accelerators and Storage Rings, Cambridge University Press (New York, 1993).

[17] G. Arduini, P. Raimondi, Transverse emittance blow-up due to injection errors, CERN SL-Note 99-022, (1999) (unpublished).

[18] E. Courant and H. Snyder, Ann. Phys. 3, 1 (1958).

[19] J. Buon, Beam Phase Space and Emittance, in CERN Yellow Report No. 94-01, (1994) (unpublished).

[20] M. Giovannozzi, Evaluation of the Emittance and Betatron Mismatch of the Old and New Continuous Transfer Beam, in preparation.

[21] J. S. Berg and F. Ruggiero, Landau damping with two-dimensional betatron tune spread, CERN-SL (AP) 1996-71, (1996) (unpublished).

[22] J. S. Berg, F. Ruggiero, Stability diagrams for Landau damping, CERN-LHC Project Report 121, (1997) (unpublished).

[23] A. W. Chao, M. Tigner, Handbook of Accelerator Physics and Engineering, World Scientific (Singapore, 1999).

[24] A. Bazzani et al., CERN Yellow Report No. 94-02, (1994) (unpublished).

[25] D. Bortolotti, M. Giovannozzi, G. Servizi, E. Todesco, and M. N. Vrahatis, Int. J. of Mod. Phys. C 6, 651 (1995). 
[26] R. Cappi, M. Giovannozzi, M. Martini, Some scenarios for preliminary tests of the new CT extraction, CERN PS Note 2002-130 (AE) (2002) (unpublished). 